\title{
Short-term effects of pamidronate on bone turnover: Can bone markers be considered predictive of the analgesic response?
}

\author{
A. MARTINETTI ${ }^{1}$, C. RIPAMONTI ${ }^{2}$, R. MICELI ${ }^{3}$, E. SEREGNI ${ }^{4}$, \\ L. MARIANI ${ }^{3}$, F. DE CONNO ${ }^{2}$, E. BAJETTA ${ }^{1}$ and E. BOMBARDIERI ${ }^{4}$ \\ ${ }^{1}$ Medical Oncology Operative Unit II, ${ }^{2}$ Day Hospital and Out-patient Clinic of Pain Therapy and Palliative Care Unit, \\ ${ }^{3}$ Medical Statistics and Biometry Unit, ${ }^{4}$ Nuclear Medicine Unit, National Cancer Institute of Milano, Milano, Italy
}

Received November 21, 2006; Accepted December 28, 2006

\begin{abstract}
Few data are available on the ability of bone markers to predict the symptomatic response to bisphosphonate therapy in patients with painful bone metastases. We evaluated the levels of bone markers in patients with bone metastases receiving pamidronate and determined the corresponding analgesic response. Forty-two patients were administered two two-week cycles of intravenous pamidronate $60 \mathrm{mg} /$ week with a three-week interval in between. Serum levels of bone formation, resorption and other bone-associated markers (osteoprotegerin, osteopontin and calcium) were measured. Levels of two urinary markers were also measured and the intensity of pain and analgesic drug consumption evaluated. A mixed effects linear modelling approach was adopted to account for possible correlation among marker levels and time on study or analgesic response. We created an indicator variable that classified the patients' analgesic response as 'improved/ stationary' or 'worsened' determined by patient reported intensity of pain and analgesic drug consumption. Eighteen patients 'worsened' and 24 were 'improved/stationary'. The results of the mixed effects models for testing the association between marker levels and time on study or analgesic response showed: i) the changes in marker levels over time did not significantly differ between the two groups; ii) the overall test for time on study was not statistically significant for C-terminal telopeptide of type I collagen (ICTP), osteoprotegerin and osteopontin; iii) in contrast, ICTP and osteoprotegerin were significantly associated with analgesic response. Biochemical markers of bone turnover, in particular ICTP and osteoprotegerin seem promising for predicting and objectively assessing the analgesic response to pamidronate treatment.
\end{abstract}

Correspondence to: Dr Carla Ripamonti, Rehabilitation and Palliative Care Operative Unit, National Cancer Institute of Milano, via Venezian 1, Milano 20133, Italy

E-mail: carla.ripamonti@istitutotumori.mi.it

Key words: bone metabolism markers, pamidronate, bone metastases, cancer-related pain, analgesic drugs

\section{Introduction}

Bone metastases are a major complication of several solid cancers, occurring in up to $70 \%$ of patients with advanced breast or prostate cancer and in approximately $15-30 \%$ of patients with carcinoma of the lung, colon, stomach, bladder, uterus, rectum, thyroid or kidney. Although bone metastases can be clinically silent, in most cases they lead to serious sequelae such as pain, fractures, spinal cord compression and hypercalcemia (1). These events often complicate the clinical course of cancer, reduce performance status and worsen patient quality of life. The conventional treatment of metastatic bone disease requires a multidisciplinary approach, including radiotherapy to the painful area, systemic treatment (e.g., hormone therapy or chemotherapy) and analgesic therapy.

In the last ten years, bisphosphonates have emerged as a valuable addition to the range of treatments for metastatic bone cancer. In fact, a number of controlled studies have shown that bisphosphonates, in particular intravenous disodium pamidronate, zoledronic acid and ibandronate can reduce the onset of skeletal complications (2-6) while also displaying significant analgesic effects (7-10).

In patients with bone metastases, an accurate assessment of how the patient and the bone metastases respond to treatment is particularly important. However, an objective evaluation of bone lesions is difficult to achieve because radiological changes are often slow and sometimes ambiguous. In recent years, circulating biochemical markers have been proposed for the investigation of bone turnover because of their accuracy in assessing dynamic changes, including the resorption and formation phases in bone remodelling. Markers of bone formation include bone-specific alkaline phosphates and procollagen peptides, while $\mathrm{N}$ - or C-terminal telopeptides of collagen I are markers of bone resorption. These biochemical markers of bone turnover appear to correlate with the presence and the extent of skeletal metastases, and have been shown to be able to predict clinical response to antiresorptive therapy with bisphosphonates (11). In contrast, little is known about the substances involved in osteoclastogenesis, such as osteoprotegerin (OPG) and osteopontin (OPN). OPG is a potent antiresorptive molecule that acts as a decoy receptor by blocking the interaction of RANKL (receptor activator of $\mathrm{NF}-\kappa \mathrm{B}$ ligand) with its functional receptor RANK, thereby inhibiting osteoclastogenesis (12). OPN is a calcium-binding 
phosphoprotein that is believed to play a role in several different and apparently distinct cellular processes. Its regulation is complex at both the cellular and molecular levels, and various hormones and growth factors have been shown to influence its production (13). OPN is also known to be an important component of cell adhesion interaction, possibly mediated by the highly conserved glycine-arginineglycine-aspartic acid-serine (GRGDS) amino acid motif found on a number of proteins that play a role in cell bond (13). Moreover, OPN appears to be an important component in the communication between osteoclasts and osteoblasts and there is strong evidence for the involvement of OPN in the formation, migration and attachment of osteoclasts and in their resorptive activity (13).

In this study we evaluated levels of bone turnover and bone associated markers [osteocalcin (BGP), bone alkaline phosphatase (BAP), N-terminal propeptide of type I procollogen (PINP), C- and N-terminal telopeptides of type I collagen (ICTP and NTx), deoxypiridinoline (D-PYR) OPG and OPN] and analgesic response in patients with metastatic bone lesions treated with intravenous pamidronate for seven weeks.

\section{Patients and methods}

All patients with any primary cancer and at least one painful bone metastasis documented on plain radiograph(s) referred to the Day Hospital and Out-patient Clinic of the Palliative Care Unit for symptom control were included in the study. All patients gave their informed consent to participate in the study. The study was carried out in the daily clinical oncological practice, where bisphosphonates are administered concomitantly to specific anticancer therapies and analgesic drugs (3). Patients were excluded if they had previously received calcitonin or any kind of bisphosphonate therapy.

The patients received two 2 -week cycles of intravenous pamidronate $60 \mathrm{mg} /$ week, with a 3-week interval in between (6 infusions over 7 weeks), followed by 1 infusion every 3 weeks for a total of 24 infusions (9). This new pamidronate infusion schedule was adopted following clinical observation and interviews with patients who had previously received intravenous pamidronate at $90 \mathrm{mg}$ every 3-4 weeks or $120 \mathrm{mg}$ every 4 weeks. These patients reported a significant increase in pain for 1-3 days after the infusion requiring an increased dose of analgesic or a switch to a stronger analgesic for pain control. Moreover, patients reported that the pain increased before the next infusion suggesting that the 'analgesic benefit' of pamidronate therapy lasted $<3$ weeks. We therefore decided to use pamidronate $60 \mathrm{mg}$ instead of the recommended 90 or $120 \mathrm{mg}$ and to shorten the interval between the doses at the beginning of the treatment, but then continue the infusions every 21 days as recommended.

Before starting pamidronate treatment (baseline), at the end of the first cycle (T1), the start of the second cycle (T2), and the end of the second cycle (T3), blood and urine samples were collected and the following clinical parameters assessed for each patient (Fig. 1): i) pain intensity [using the Likert verbal scale (no pain, a little, much, very much)]; ii) type of analgesic drugs used (according to the three-step WHO analgesic ladder) (14); iii) the frequency of NSAID administration

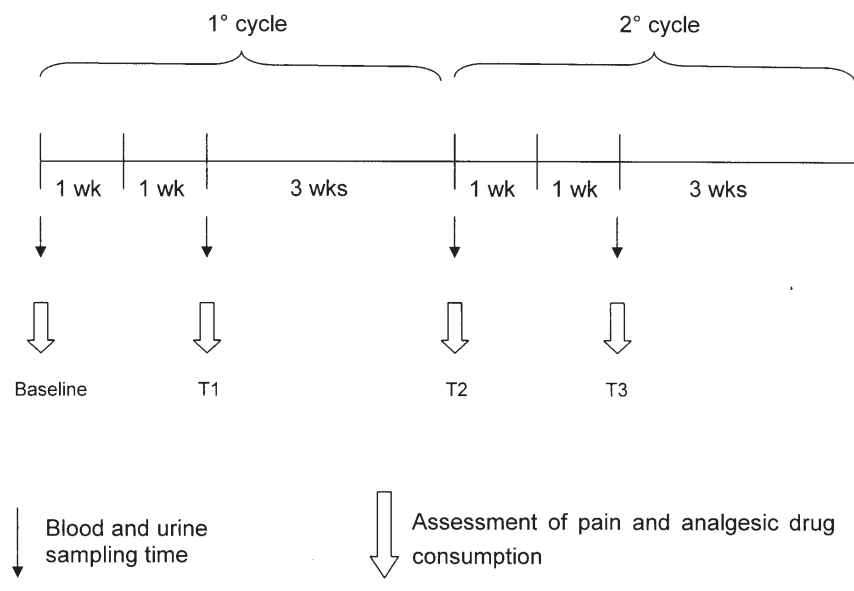

Figure 1. Infusion schedule of pamidronate and assessment times.

either alone, or in association with a regular dose of opioid; iv) doses of opioid drugs as equivalent of the daily dosage of oral morphine (EDDOM). Blood samples were collected between 9:00 and 10:00 a.m. after a 12-h fast and before pamidronate administration. Serum was separated by centrifugation immediately after clotting and stored at $-70^{\circ} \mathrm{C}$ until measurement. Urine samples were obtained from the second void of the morning and were also stored at $-70^{\circ} \mathrm{C}$ until assay.

All biological measurements were performed at the laboratory of the Nuclear Medicine Unit. Levels of three serum markers of bone formation (PINP, BGP and BAP) were evaluated.

As an indicator of bone resorption, levels of ICTP and two urinary markers (D-PYR and NTx) were assessed. Circulating levels of the bone associated markers OPG, OPN and calcium were evaluated.

Serum levels of PINP were measured using the radioimmunoassay kit PINP RIA (Orion Diagnostic); the intraand inter-assay coefficients of variations were 7.2 and $4.6 \%$, respectively. The analytical sensitivity was $2 \mu \mathrm{g} / \mathrm{l}$. The normal range was $19-84 \mu \mathrm{g} / 1$.

Serum levels of BGP and B-ALP were measured using the immunoenzymatic assays Novocalcin and Alkaphase-ALP (Metra Biosystem), respectively. The intra- and inter-assay coefficients of variations were $<7 \%$ for both tests. The analytical sensitivity of the Novocalcin assay was $0.45 \mathrm{ng} / \mathrm{ml}$; the BGP normal range was $3.7-10.0 \mathrm{ng} / \mathrm{ml}$. The analytical sensitivity of the Alkaphase-ALP assay was $0.7 \mathrm{U} / 1$ and the normal range was 14-42 U/1.

ICTP was measured in serum using the radioimmunoassay kit ICTP RIA (Orion Diagnostic), the intra- and inter-assay coefficients of variations were 4.2 and $5.6 \%$, respectively. The ICTP analytical sensitivity was $0.5 \mu \mathrm{g} / \mathrm{l}$, and the normal range was $1.8-5.0 \mu \mathrm{g} / 1$.

Urine NTx and D-PYR levels were determined by the Osteomark (Ostex International) and the D-Pyr (Metra Biosystem) assays kits, respectively. The intra- and interassay coefficients of variations were $<6.5 \%$ for both tests. NTx values were corrected for urinary dilution by creatinine analysis and reported as nanomole of bone collagen equivalent per millimole of creatinine ( $\mathrm{nMBCE} / \mathrm{mM}$ creatinine). The Osteomark test analytical sensitivity was $20 \mathrm{nMBCE}$, and 
normal range was $565 \mathrm{nMBCE}$. D-PYR values were corrected for urinary dilution by creatinine analysis and reported as $\mathrm{nM} / \mathrm{mM}$ creatinine. The D-PYR test analytical sensitivity was $2 \mathrm{nM}$ and normal range was 3.0-7.4 nM/mM creatinine.

OPG was measured in serum using the OPG ELISA produced by DRG Gmbh (Germany) and purchased from TEMA ricerca s.r.l. (Italy). This assay is a sandwich-type ELISA for the direct determination of OPG in serum using two highly specific antibodies against OPG (homodimeric and monomeric forms). The binding antibody is attached to the wells of the microplate and the detection antibody is labelled with biotin.

Serum levels of OPN were measured using the enzyme immunometric assay kit produced by Assay Designs Inc (USA) purchased from TEMA ricerca s.r.l. The polyclonal antibody in this assay is immobilised on a microtiter plate and binds OPN present in the sample. Further detection occurs using a second monoclonal antibody labelled with horseradish peroxidase. Calcium serum levels were evaluated by a calorimetric assay using a Roche automated clinical chemistry analyser.

Statistical methods. We analysed the relationship between bone markers, time on study and analgesic response. The main analysis focused on the variation of bone marker levels during the observation time. We considered the times at which information about bone metastasis progression was available: baseline, T1, T2 and T3. A mixed effects linear modelling approach (15) was adopted, to account for possible correlation among marker longitudinal measurements within the same patient. In each model the dependent variable was the marker. To approximate a normal distribution, a logarithmic transformation was needed for all markers except calcium and OPG. Model adjustment was performed for covariates presenting variations over time, including: i) presence or absence of radiotherapy; ii) bone metastasis progression; iii) analgesic response as assessed by the Likert scale and drug consumption in terms of EDDOM. At each time-point, the pain intensity was expressed as change from the previous assessment: -1 (less pain), 0 (no variation) and +1 (more pain). A timeindependent categorical variable was then created based on the algebraic sum of variations over time; the categories were: 'reduced pain' (sum <0), 'no variation' (sum =0) and 'increased pain' (sum $>0$ ). The drug consumption variation with respect to the previous assessment was expressed as: -1 (reduced consumption), 0 (no variation) and +1 (increased consumption) separately for WHO analgesic ladder 1 st step (NSAIDs alone) and WHO analgesic ladder 2nd and 3rd step (weak opioid \pm NSAIDs and strong opioid \pm NSAIDs). A time-independent categorical variable was then created based on the algebraic sum of variations; the categories were: 'reduced consumption' (sum $<0$ on WHO analgesic ladder 1 st step and sum $=0$ on WHO analgesic ladder $2 \mathrm{nd}+3 \mathrm{rd}$ step or sum $<0$ on WHO analgesic ladder 2 nd +3 rd step), 'no variation' ( sum $=0$ on both WHO analgesic ladder 1 st step and 2nd + 3rd step) and 'increased consumption' (all other cases). The categories of the variable patient analgesic responses were: 'worsened' (increased pain intensity or drug consumption) or 'improved/ stationary'.

All the three above-defined covariates were entered into the models as fixed effects, together with the interaction
Table I. Patient demographics and disease characteristics.

\begin{tabular}{|c|c|c|}
\hline & No. & $(\%)$ \\
\hline Total & 42 & - \\
\hline \multicolumn{3}{|l|}{ Age (years) } \\
\hline Median & 61 & \\
\hline Range & $33-78$ & \\
\hline \multicolumn{3}{|l|}{ Sex } \\
\hline Female & 35 & 83 \\
\hline Male & 7 & 17 \\
\hline \multicolumn{3}{|c|}{ Site of primary tumour } \\
\hline Breast & 33 & 79 \\
\hline Rectum & 3 & 7 \\
\hline Thyroid & 2 & 4 \\
\hline Other & 4 & 10 \\
\hline \multicolumn{3}{|l|}{ No. of lesions } \\
\hline Single lesion & 14 & 33 \\
\hline $2-3$ & 18 & 43 \\
\hline$>3$ & 10 & 24 \\
\hline \multicolumn{3}{|l|}{ Therapy } \\
\hline None & 12 & 29 \\
\hline Chemotherapy & 8 & 19 \\
\hline Hormonotherapy & 22 & 52 \\
\hline \multicolumn{3}{|c|}{ Pain intensity (Likert scale) } \\
\hline No pain & 2 & 5 \\
\hline A little & 22 & 52 \\
\hline Much & 18 & 43 \\
\hline Very much & 0 & 0 \\
\hline
\end{tabular}

Karnofsky performance status

$\begin{array}{rrr}50 & 2 & 4 \\ 60 & 8 & 20 \\ 70 & 26 & 62 \\ 80 & 6 & 14\end{array}$

terms time $\mathrm{x}$ analgesic response; the latter to test whether changes over time in marker levels differed for 'worsened' and 'improved/stationary' patients. When the overall test for the time effect was significant, the following comparisons among marker means were performed: i) baseline vs. time $\mathrm{T} 1$; ii) $\mathrm{T} 1 \mathrm{vs}$. $\mathrm{T} 3$ to investigate if the marker values at the end of each treatment cycle levelled out; iii) when the latter test was not significant T2 vs. T1/T3 to have more insights over the time trend. In all the mixed models we adopted unstructured correlation structures and, as commonly suggested, the Restricted Maximum Likelihood (REML) estimation algorithm.

To integrate the analysis described above, we investigated the marker ability to predict analgesic response. This was done by means of logistic regression analysis considering ICTP, OPG and OPN which were clearly associated with the 
Table II. Analgesic treatment at baseline.

\begin{tabular}{lrc}
\hline WHO analgesic ladder & No. & $(\%)$ \\
\hline No analgesic drug & 5 & 11.9 \\
1st step (NSAIDs alone) & 12 & 28.6 \\
2nd step (weak opioids \pm NSAIDs) & 15 & 35.7 \\
3rd step (strong opioids \pm NSAIDs) & 10 & 23.8 \\
\hline
\end{tabular}

analgesic response and stable over time. In the models, marker data were synthesized by calculating the mean per-patient values; the latter were modelled by linear terms or, alternatively, in a flexible way by 3 -knots restricted cubic splines (16), selecting the model with the lower value of the Akaike Information Criterion (Akaike H, Proc 2nd Int Symp on Information Theory, Budapest, 1973). As a measure of model predictive ability we used the area under the Receiver Operating Curve (AUC-ROC), estimated by the Harrell C statistic (17) bootstrap-corrected (bias corrected) for over optimism. SAS ${ }^{\mathrm{TM}}$ software (SAS Institute Inc., Cary, North Carolina, 2000) was used to perform the modelling and statistical calculations. Two-sided p-values below the 5\% conventional threshold were considered significant.

\section{Results}

Forty-two patients with advanced cancer and bone metastases entered the study. Patient demographics and disease characteristics are presented in Table I, and Table II shows the analgesic treatment at baseline according to the WHO guidelines. No patient received radiotherapy before the beginning of the study.

Ten patients started radiotherapy during the 2 nd treatment cycle and only half of them continued radiotherapy until the end of the 2 nd cycle. During the observation period 18 patients (43\%) 'worsened' (showed increased pain intensity or drug consumption); these patients did not differ from the 24 patients in the 'improved/stationary' category with respect to the number of lesions.

The geometric means and the $95 \%$ respective confidence intervals for each bone marker analysed are presented separately for the two analgesic response categories in Table III; a pictorial representation is given in Fig. 2. For all the bone markers, in the 'worsened' group the baseline means were greater than those in the 'improved/stationary' group.

Mean levels of ICTP and OPG in serum did not show any variation with time, maintaining a constant difference between the two categories of analgesic response. Serum levels of OPN were almost stable over time, showing a slight reduction from baseline to T1 in only the 'improved/stationary' group. Similar reductions over time were observed, especially from baseline to T1, for the other bone markers (BAP, BGP, PINP, PYR, NTx and $\mathrm{Ca}$ ) in patients in both analgesic response categories.

Table IV shows the results of the mixed effects models in terms of p-values for testing the association between bone marker levels and time on study or analgesic response. Radio- therapy administration and bone metastasis progression were not significantly associated with bone marker levels in any model (data not shown). During treatment, the changes in bone marker levels over time were not significantly different for 'worsened' and 'improved/stationary' patients, as indicated by the test for interaction time $\mathrm{x}$ analgesic response. Thus, in Table IV we reported the results of the models without the interaction terms. For ICTP, OPG and OPN the overall test for time of study was not statistically significant (second column of Table IV). ICTP and OPG were significantly associated with analgesic response, and a borderline p-value of 0.079 was achieved by OPN. These results for the above mentioned three bone markers are consistent with the time pattern of means shown in Fig. 2. The overall test for the time of study was significant for all of the remaining markers, with a borderline p-value of 0.056 for BAP. Investigating further, a significant reduction from baseline to the end of the first cycle (baseline vs. T1) was observed for BAP, BGP, PINP and PYR. The bone marker levels may be considered stable thereafter as indicated by no significant association between markers and time of study for T1 vs. T3 and T2 vs. T1/T3.

Significant results were obtained for both calcium and NTx, for baseline vs. T1 and T1 vs. T3. In both categories of analgesic response, calcium levels decreased at the end of the first cycle followed by a slight increase thereafter, whereas in the 'worsened' group NTx levels decreased at the end of the first cycle and remained stable thereafter, and in the 'improved/ stationary' group NTx levels remained stable (Table III).

Among the markers that were investigated for their ability to predict analgesic response, the best performance was achieved by ICTP (AUC-ROC=0.80, Fig. 3), followed by OPG (AUC-ROC=0.72) and OPN (AUC-ROC=0.71). Even for ICTP, the AUC-ROC figure obtained implied some tradeoff between sensitivity and specificity. For instance, to achieve a specificity of $\sim 90 \%$, the sensitivity was $25 \%$; conversely, with a sensitivity of $92 \%$, the specificity was $\sim 40 \%$.

\section{Discussion}

It is generally accepted that bisphosphonates can provide relief of bone pain $(5,7,10)$ in about $50 \%$ of patients $(11)$. The reasons for a lack of symptomatic response in the other $50 \%$ of patients are not clear. Bone pain, as a result of bone metastases, is a complex process produced by mechanical factors and focal tumour-induced osteolysis. Moreover, the inflammatory and immunological reactions produce the chemical mediators that increase pain perception such as prostaglandins, substance $\mathrm{P}$, bradykinins, interleukins and tumour necrosis factors.

Various studies have shown that pamidronate is effective in reducing pain scores and/or analgesic consumption in patients with bone metastases $(7,18)$. Pamidronate is a potent inhibitor of bone resorption acting through various mechanisms. Ultrastructural examination of the osteoclasts of animals treated with bisphosphonates has shown a reduction in the volume of the septate border (the site of osteoclastic bone resorption) and abnormalities in the structure as well as in the enzymatic activity of lysosomes (19).

Recently, particular interest has been focused on the use of biochemical markers of bone metabolism as an alternative 
Table III. Descriptive statistics of marker distribution in the two categories defined by analgesic response.

\begin{tabular}{|c|c|c|c|c|c|}
\hline \multirow[b]{2}{*}{ Marker } & \multirow[b]{2}{*}{ Time } & \multicolumn{2}{|c|}{ Worsened group } & \multicolumn{2}{|c|}{ Improved/stationary group } \\
\hline & & M & $95 \% \mathrm{CI}$ & M & $95 \% \mathrm{CI}$ \\
\hline \multirow[t]{4}{*}{ BAP U/1 } & Baseline & 35.9 & $27.3-47.2$ & 26.1 & $23.0-29.7$ \\
\hline & $\mathrm{T} 1$ & 33.3 & $25.8-43.1$ & 24.1 & $19.9-29.1$ \\
\hline & $\mathrm{T} 2$ & 31.8 & $23.3-43.4$ & 22.9 & $18.2-28.7$ \\
\hline & $\mathrm{T} 3$ & 31.0 & $22.6-42.4$ & 22.1 & $17.3-28.1$ \\
\hline \multirow[t]{4}{*}{ BGP ng/ml } & Baseline & 3.9 & $2.6-5.9$ & 3.6 & $2.7-4.9$ \\
\hline & $\mathrm{T} 1$ & 3.1 & $2.1-4.6$ & 3.3 & $2.2-4.7$ \\
\hline & $\mathrm{T} 2$ & 2.4 & $1.5-3.9$ & 3.3 & $2.2-4.8$ \\
\hline & $\mathrm{T} 3$ & 2.5 & $1.6-4.0$ & 2.8 & $1.9-4.1$ \\
\hline \multirow[t]{4}{*}{ Calcium mg/dl } & Baseline & 9.6 & $9.2-9.9$ & 9.2 & $8.9-9.4$ \\
\hline & $\mathrm{T} 1$ & 9.0 & $8.8-9.2$ & 8.8 & $8.6-8.9$ \\
\hline & $\mathrm{T} 2$ & 9.1 & $8.9-9.3$ & 9.1 & $8.9-9.3$ \\
\hline & $\mathrm{T} 3$ & 9.2 & $8.9-9.4$ & 9.1 & $8.9-9.3$ \\
\hline \multirow[t]{4}{*}{ ICTP $\mu \mathrm{g} / 1$} & Baseline & 10.3 & $7.7-13.8$ & 6.4 & $5.5-7.5$ \\
\hline & $\mathrm{T} 1$ & 10.9 & $8.4-14.3$ & 6.3 & $5.3-7.6$ \\
\hline & $\mathrm{T} 2$ & 11.4 & 9.4-13.9 & 6.7 & $5.4-8.4$ \\
\hline & T3 & 11.1 & $9.0-13.8$ & 6.8 & $5.4-8.5$ \\
\hline \multirow{4}{*}{$\begin{array}{l}\text { NTX nmol BCE/ } \\
\text { mmol crea }\end{array}$} & Baseline & 177.5 & 103.6-303.9 & 130.7 & $100.0-170.9$ \\
\hline & $\mathrm{T} 1$ & 49.2 & $33.4-72.6$ & 48.6 & $26.9-53.0$ \\
\hline & $\mathrm{T} 2$ & 65.9 & $44.0-99.0$ & 37.7 & $35.8-65.9$ \\
\hline & $\mathrm{T} 3$ & 66.8 & $49.1-91.0$ & 45.5 & $32.8-63.2$ \\
\hline \multirow[t]{4}{*}{ OPG pmol/1 } & Baseline & 5.1 & $4.0-6.2$ & 4.5 & $3.7-5.4$ \\
\hline & $\mathrm{T} 1$ & 5.5 & $3.9-7.0$ & 4.4 & $3.7-5.0$ \\
\hline & $\mathrm{T} 2$ & 5.5 & $4.5-6.6$ & 4.2 & $3.7-4.7$ \\
\hline & $\mathrm{T} 3$ & 5.2 & $4.5-6.0$ & 4.1 & $3.3-5.0$ \\
\hline \multirow[t]{4}{*}{ OPN $\mu \mathrm{g} / 1$} & Baseline & 29.4 & $22.9-37-6$ & 25.7 & $19.1-34.6$ \\
\hline & $\mathrm{T} 1$ & 33.4 & $24.3-45.7$ & 22.5 & $16.7-30.5$ \\
\hline & $\mathrm{T} 2$ & 33.6 & $23.5-48.0$ & 19.4 & $14.5-25.9$ \\
\hline & $\mathrm{T} 3$ & 35.0 & $25.8-47.3$ & 21.4 & $16.1-28.6$ \\
\hline \multirow[t]{4}{*}{$\mathrm{PINP} \mu \mathrm{g} / \mathrm{l}$} & Baseline & 88.0 & 59.7-129.6 & 67.2 & $85.5-46.4$ \\
\hline & $\mathrm{T} 1$ & 63.5 & 42.9-93.9 & 52.3 & $69.2-37.0$ \\
\hline & $\mathrm{T} 2$ & 78.4 & $52.5-117.1$ & 50.0 & $65.6-31.5$ \\
\hline & T3 & 67.5 & 44.8-101.7 & 42.1 & $60.0-23.2$ \\
\hline \multirow{4}{*}{$\begin{array}{l}\text { PYR nM/ } \\
\text { mmol crea }\end{array}$} & Baseline & 20.6 & $16.4-25.8$ & 18.6 & $15.4-22.4$ \\
\hline & $\mathrm{T} 1$ & 13.9 & $11.0-17.4$ & 12.0 & $9.4-15.3$ \\
\hline & $\mathrm{T} 2$ & 16.4 & $13.3-20.3$ & 13.9 & $11.4-17.0$ \\
\hline & $\mathrm{T} 3$ & 16.9 & $13.9-20.5$ & 14.5 & 10.9-19.1 \\
\hline
\end{tabular}

Worsened group, patients with increase in pain intensity or drug consumption during follow-up; improved/stationary group, all other patients. M, geometric mean; arithmetic mean for calcium and OPG. T1, end of first cycle; T2, start of second cycle; T3, end of second cycle. CI, confidence interval.

instrument to evaluate the response to bisphosphonate treatment.

In this study we measured a selection of bone metabolism and bone-associated markers in 42 cancer patients treated with 6 intravenous infusions of pamidronate $60 \mathrm{mg}$ within 7 weeks.

We have verified that during pamidronate therapy, ICTP, OPG and OPN serum levels did not significantly change in 

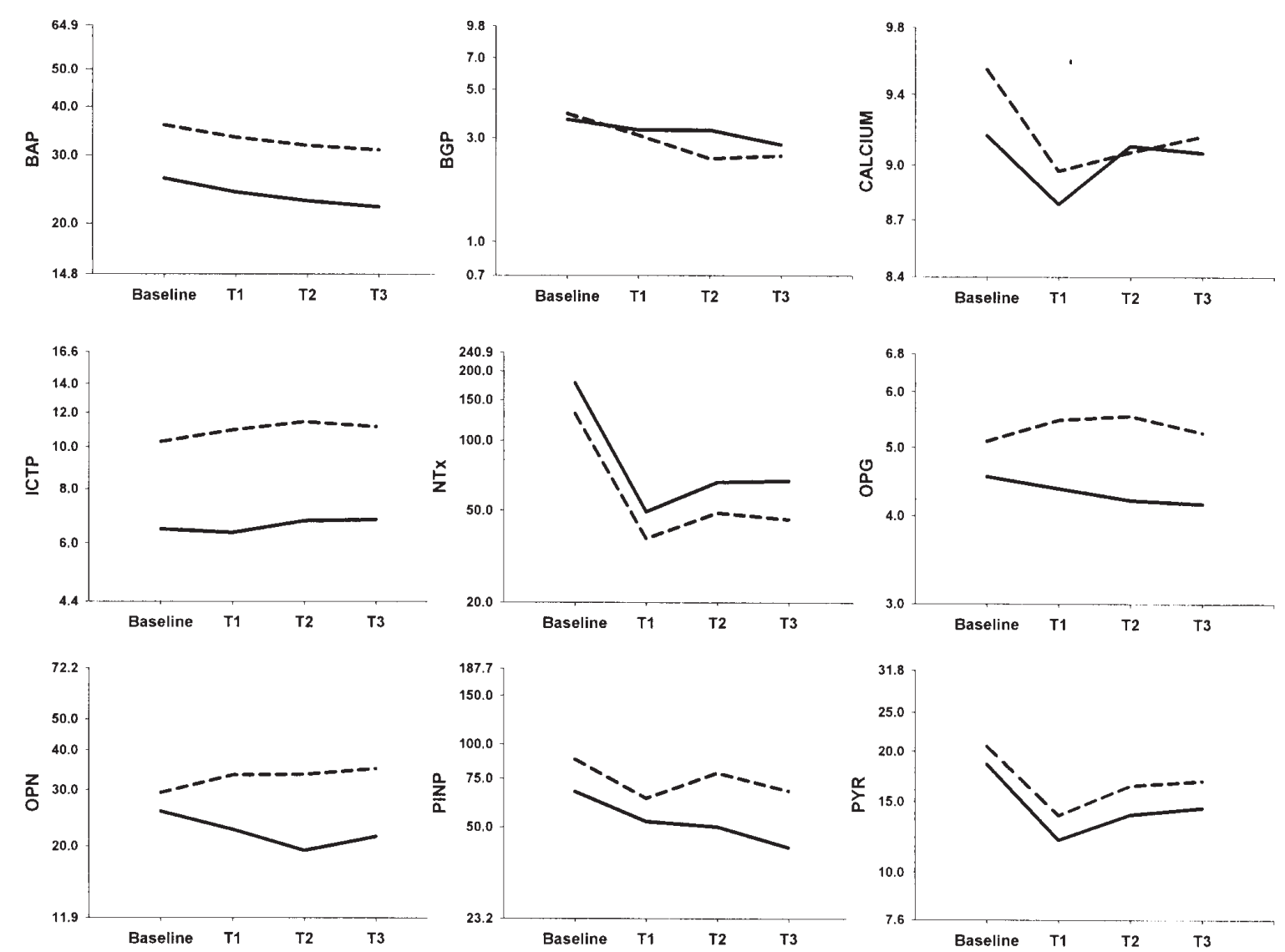

Figure 2. Representation in logarithmic scale of marker geometric means at various times during the study in the two categories defined by analgesic response. The vertical axis limits correspond, respectively, to the 10th and 90th centiles of the marker distribution. T1, end of first cycle; T2, start of second cycle; T3, end of second cycle. Dashed line, worsened group (patients with increase in pain intensity or drug consumption during the study period); continued line, improved/stationary group (all other patients).

Table IV. p-values for testing the association between marker levels and time of study or analgesic response. ${ }^{\mathrm{a}}$

\begin{tabular}{lccccc}
\hline & \multicolumn{3}{c}{ Time } & Tnalgesic \\
\cline { 2 - 6 } Marker & Overall & Baseline vs. T1 & T1 vs. T3 & T2 vs. T1/T3 & $\begin{array}{c}\text { Ansense } \\
\text { response }\end{array}$ \\
\hline BAP & 0.056 & 0.024 & 0.334 & 0.636 & 0.119 \\
BGP & 0.048 & 0.014 & 0.314 & 0.762 & 0.465 \\
Calcium & 0.001 & 0.042 & 0.005 & - & 0.412 \\
ICTP & 0.712 & - & - & - & 0.001 \\
NTX & $<0.001$ & $<0.001$ & -0.017 & - & 0.184 \\
OPG & 0.703 & - & - & - & 0.028 \\
OPN & 0.621 & - & - & - & 0.079 \\
PINP & $<0.001$ & $<0.001$ & 0.1386 & 0.224 & 0.411 \\
PYR & $<0.001$ & 0.013 & & 0.388 & 0.242 \\
\hline
\end{tabular}

${ }^{a}$ p-values at $\mathrm{F}$ test obtained from mixed effects linear models (see Statistical methods section). T1, end of first cycle; T2, start of second cycle; $\mathrm{T} 3$, end of second cycle.

either those patients with an improved analgesic response or in those with a worsening response. All other bone markers showed a secondary reduction, more evident after the first two weeks of therapy (during the 1 st cycle when no patient was on radiotherapy). However, during treatment, changes in bone marker levels over time did not significantly differ for 'worsened' or 'improved/stationary' patients, as indicated by the test for interaction time $\mathrm{x}$ analgesic response. 


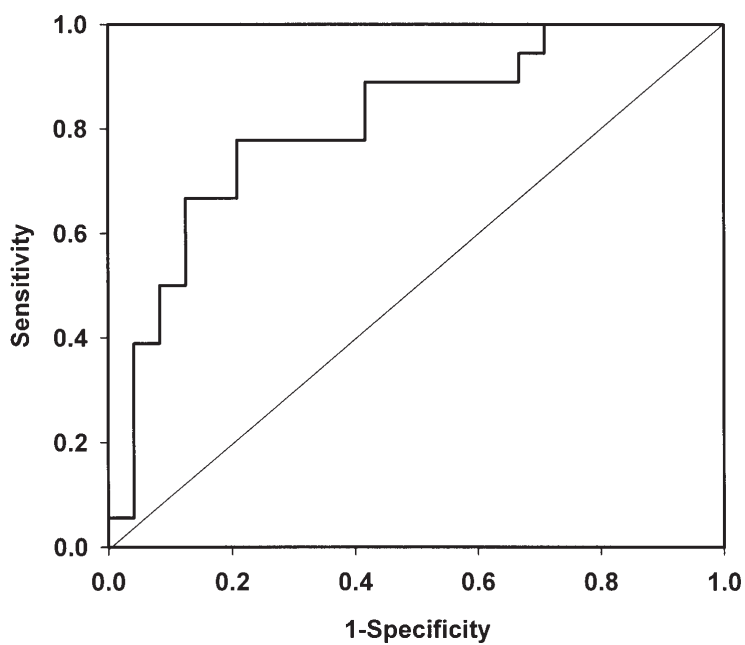

Figure 3. Receiver operating curve of ICTP across levels of probability of being improved or stationary, as estimated by the logistic model (AUC$\mathrm{ROC}=0.80$ ).

Although the levels of the bone markers at baseline and during treatment were generally greater in the 'worsened' group compared with the 'improved/stationary' group (Table III), the difference was statistically significant for ICTP and OPG and of borderline significance for OPN (Table IV). When the ability to predict analgesic response was specifically investigated, ICTP showed the best capability (AUC-ROC $=0.80$ ), followed by OPG and OPN (AUC-ROC $=0.72$ and 0.71 , respectively). Berutti et al (20) showed that in 323 patients with bone metastases from various primary malignancies, ICTP was the only marker correlated with bone pain independent of the primary cancer. This could indicate that the bone metabolism markers have a different behaviour, particularly between ICTP and NTx, in relation to both their variation during treatment and their ability to predict analgesic response.

However, future ad hoc studies, much larger than the present one, should be conducted to investigate if these three bone markers could be used to predict the analgesic effect of pamidronate.

Costa et al (21) reported that ICTP is unaffected by bisphosphonate therapy while urine NTx levels are reduced during bisphosphonate therapy. A possible explanation for these results is the fact that these bone markers are the result of different events. NTx is related to the inhibition of osteoclastic activity and therefore is an indicator of the pamidronate pharmacological mechanism of action. In contrast, ICTP appears to represent a bone collagen product derived from an osteoclast-independent mechanism of bone degradation (21) that is not the cellular target of pamidronate. This would explain why the ICTP levels remain unchanged during pamidronate treatment. The ability of ICTP to predict the analgesic effect of pamidronate can be linked to the fact that in patients with low ICTP levels, and therefore a low tumoural burden, pain is the result of the osteoclastic pathway activity. This may be the reason why a drug, specific in blocking the osteoclast activity such as pamidronate is active in controlling pain. In contrast, in patients with high ICTP levels, and greater tumoural burden, the intensity of pain is primarily due to the tumour compression/infiltration of the sensitive areas rather than osteoclastic pathway activity; in this case, pamidronate would play only a partial analgesic role.

Some authors have shown that those patients who do not derive a clinical benefit from pamidronate therapy have high bone turnover, with high levels of NTx that do not normalise during treatment (22-24). According to Lipton et al (22) the goal of pamidronate therapy should be to normalise NTx excretion. However, bone turnover in patients with high NTx levels is also increased by factors produced by the tumour itself (e.g., proteases) that pamidronate is unable to control.

All our patients had a significant reduction of NTx levels during pamidronate treatment, however, only 24 patients (57\%) reported an analgesic benefit. The two principal pathways for bone resorption are modulated by two proteases: cathepsin $\mathrm{K}$ and metalloproteinases (MMPs). Cathepsin $\mathrm{K}$ is the main enzyme involved in bone resorption, while MMPs become the predominant enzymes in particular cases, such as the presence of a bone metastases $(25,26)$. Pamidronate is able to inhibit bone resorption modulated by cathepsin $\mathrm{K}$, but not MMPs. It has been demonstrated that tumoural cells release large quantities of MMPs and the pathway modulated by MMPs results in an increase in the levels of ICTP (25). This may be a reason for the lack of analgesic response to pamidronate in those patients with high ICTP levels. Likewise, high levels of OPG and OPN (borderline) are predictors of lack of analgesic response to pamidronate, as these bone markers can be produced by the tumour itself to facilitate its spread.

The fact that pain relief occurs in about $50 \%$ of patients treated with bisphosphonates (11) has encouraged attempts to identify patients with bone metastases at an early stage in order to start appropriate treatment, monitor the effectiveness of the chosen regimen, and predict which patients will need a more specific analgesic therapy.

In conclusion, bone markers, in particular ICTP and OPG, offer the possibility to reliably predict and objectively assess the analgesic response to pamidronate treatment. Using these bone markers we are able to identify factors underlying resistance to pamidronate analgesic efficacy, such as a higher rate of bone resorption as determined by higher ICTP and OPG baseline levels. The measurement of bone markers may be of particular value when the aim is not only the prevention of skeletal complications or progression of the disease but also to prevent or treat bone metastasis-related pain.

\section{Acknowledgements}

The authors thank Ms. Nadia Crose for establishing the data base.

\section{References}

1. Body JJ: Effectiveness and cost of bisphosphonates therapy in tumour bone disease. Cancer 97 (suppl 3): 859-865, 2003.

2. Berenson JR, Hillner BE, Kyle RA, et al: American Society of Clinical Oncology Clinical Practice Guidelines: the role of bisphosphonates in multiple myeloma. J Clin Oncol 20: 3719-3736, 2002.

3. Hillner BE, Ingle JN, Chlebowski RT, et al: American Society of Clinical Oncology 2003 update on the role of bisphosphonates and bone health issues in women with breast cancer. J Clin Oncol 21: 4042-4057, 2003. 
4. Small EJ, Smith MR, Seaman JJ, Petrone S and Ortu Kowalski M: Combined analysis of two multicenter, randomized, placebocontrolled studies of pamidronate disodium for the palliation of bone pain in men with metastatic prostate cancer. J Clin Oncol 21: 4277-4284, 2003

5. Saad F, Gleason DM, Murray R, et al: A randomized, placebocontrolled trial of Zoledronic Acid in patients with hormonerefractory metastatic prostate carcinoma. J Natl Cancer Inst 94: 1458-1468, 2002.

6. Body JJ, Diel IJ, Lichinitser MR, et al: Intravenous ibandronate reduces the incidence of skeletal complications in patients with breast cancer and bone metastases. Ann Oncol 14: 1399-1405, 2003.

7. Fulfaro F, Casuccio A, Ticozzi $C$ and Ripamonti C: The role of bisphosphonates in the treatment of painful metastatic bone disease: a review of phase III trials. Pain 78: 157-169, 1998.

8. Ripamonti $\mathrm{C}$ and Fulfaro F: Malignant bone pain: pathophysiology and treatments. Curr Rev Pain 4: 187-196, 2000.

9. Groff L, Zecca E, De Conno F, et al: The role of disodium pamidronate in the management of bone pain due to malignancy. Palliat Med 15: 297-307, 2001.

10. Mancini I, Dumon JC and Body JJ: Efficacy and safety of ibandronate in the treatment of opioid-resistant bone pain associated with metastatic bone disease: a pilot study. J Clin Oncol 22: 3587-3592, 2004

11. Vinholes JJ, Purohit OP, Abbey ME, Eastell R and Coleman RE: Relationship between biochemical and symptomatic response in a double-blind randomized trial of pamidronate for metastatic bone disease. Ann Oncol 8: 1243-1250, 1997.

12. Anderson DM, Maraskovsky E, Billingsley WL, et al: A homologue of the TNF receptor and its ligand enhance T-cell growth and dendritic-cell function. Nature 390: 175-179, 1997.

13. Sodek J, Ganss B and McKee MD: Osteopontin. Crit Rev Oral Biol Med 11: 279-303, 2000.

14. World Health Organization. Cancer Pain Relief. World Health Organization, Geneve, 1986

15. Searle SR, Casella G and McCulloch CE: Variance Components. John Wiley \& Sons, Inc., NY, 1992.

16. Durrleman S and Simon R: Flexible regression models with cubic splines. Stat Med 8: 551-561, 1989.
17. Harrell FE Jr, Lee KL, Califf RM, Pryor DB and Rosati RA Regression modelling strategies for improved prognostic prediction. Stat Med 3: 143-152, 1984

18. Hortobagyi GN, Theriault RL, Porter L, et al: Efficacy of pamidronate in reducing skeletal complications in patients with breast cancer and lytic bone metastases. Protocol 19 Aredia Breast cancer Study Group. N Engl J Med 335: 1785-1791, 1996.

19. Fleisch H: Bisphosphonates - mechanisms of action. In: Disodium Pamidronate (ADP) in the Treatment of Malignancy-related Disorders. Burckhardt P (ed). Hans-Huber Publishers, Toronto, pp21-35, 1988.

20. Berruti A, Dogliotti L, Gorzrgno G, et al: Differential pattern of bone turnover in relation to bone pain and disease extend in bone in cancer patients with skeletal metastases. Clin Chem 45: 1240-1247, 1999.

21. Costa L, Demers LM, Gouveia-Oliveira A, et al: Prospective evaluation of the peptide-bound collagen type I cross-links $\mathrm{N}$ telopeptide and C-telopeptide predicting bone metastases status. J Clin Oncol 20: 850-856, 2002.

22. Lipton A, Demers L, Curley E, et al: Markers of bone resorption in patients treated with Pamidronate. Eur J Cancer 34 2021-2026, 1998

23. Rosen HN, Moses AC, Garber J, Ross DS, Lee SL and Greenspan SL: Utility of biochemical markers of bone turnover in the follow-up of patients treated with bisphosphonates. Calcif Tissue Int 63: 363-368, 1998.

24. Coleman RE, Major P, Lipton A, et al: Predictive value of bone resorption and formation markers in cancer patients with bone metastases receiving the bisphosphonate zoledronic acid. J Clin Oncol 23: 4925-4935, 2005.

25. Garnero P, Ferreras M, Karsdal MA, et al: The type I collagen fragments ICTP and CTX reveal distinct enzymatic pathways of bone collagen degradation. J Bone Miner Res 18: 859-867, 2003.

26. Atley LM, Mort SJ, Lalumiere M and Eyre DR: Proteolysis of human bone collagen by catepsina $\mathrm{K}$. Characterization of the cleavage sites generating the cross-linked N-telopeptide neoepitope. Bone 26: 241-247, 2000. 